\title{
Carnets
}

Revue électronique d'études françaises de l'APEF

Première Série - 4 Numéro Spécial | 2012

Invasions \& Évasions

\section{Traduction et invasion culturelle: Jean Echenoz en portugais et José Saramago en français}

\section{Dominique Faria}

\section{(2) OpenEdition}

1 Journals

Édition électronique

URL : http://journals.openedition.org/carnets/7930

DOI : 10.4000/carnets. 7930

ISSN : 1646-7698

Éditeur

APEF

Édition imprimée

Date de publication : 1 juin 2012

Pagination : 303-315

Référence électronique

Dominique Faria, «Traduction et invasion culturelle: Jean Echenoz en portugais et José Saramago en français », Carnets [En ligne], Première Série - 4 Numéro Spécial | 2012, mis en ligne le 23 juin 2018, consulté le 23 octobre 2019. URL : http://journals.openedition.org/carnets/7930 ; DOI : 10.4000/ carnets. 7930

\section{(c) (7) 8}

Carnets est mis à disposition selon les termes de la licence Creative Commons - Atribution - Pas d'utilisation commerciale 4.0 International. 


\title{
TRADUCTION ET INVASION CULTURELLE Jean Echenoz en portugais et José Saramago en français
}

DOMINIQUE FARIA

Universidade dos Açores

dominiquefaria@uac.pt

\begin{abstract}
Résumé
La traduction est un moyen d'invasion culturelle dont le pouvoir est souvent sous-estimé. Elle permet, pourtant, l'introduction, dans une culture nationale, d'éléments appartenant à une culture étrangère. Ce pouvoir de la traduction est toutefois potentiel. Pour qu'il y ait effectivement invasion culturelle par le biais de la traduction, il faut la contribution des entités du pays envahisseur (les organismes qui font la promotion de la littérature à l'étranger) et de celles du pays envahi (maisons d'édition, traducteurs et lecteurs). Les traductions de Je m'en vais de Jean Echenoz et de História do cerco de Lisboa de José Saramago, en portugais et en français respectivement, fonctionneront comme le point de départ pour une réflexion sur le rôle de la traduction dans le rapport entre le Portugal et la France à l'époque contemporaine.
\end{abstract}

\begin{abstract}
Translation is a means of cultural invasion whose power is often underestimated. It nonetheless allows the penetration of elements of a foreign culture into a national culture. This, however, is a potential power. For cultural invasion to actually take place through translation, the entities from the invading country (the institutions which promote national literature abroad) as well as those from the invaded country (publishers, translators and readers) must contribute to it. The Portuguese translation of Je m'en vais by Jean Echenoz and the French translation of História do cerco de Lisboa by José Saramago will work as the starting point to a reflection on the role of translation in the relationship between Portugal and France in contemporary times.
\end{abstract}

Mots-clés: traduction, invasion culturelle, Saramago, Echenoz, roman contemporain.

Keywords: translation, cultural invasion, Saramago, Echenoz, contemporary novel. 
La traduction est probablement une des formes d'invasion culturelle les plus fréquentes, mais aussi, très souvent, des moins valorisées. Silencieuse et discrète, elle introduit, texte à texte, les traits de la culture de départ dans la culture d'arrivée. Ce pouvoir n'est toutefois pas inhérent à la traduction, pouvant être neutralisé ou potentialisé par la capacité qu'ont les structures du pays de départ de promouvoir la publication d'ouvrages nationaux à l'étranger, ainsi que par les stratégies éditoriales et de traduction dans le pays d'arrivée, c'est-à-dire par la résistance que la culture d'arrivée offre à l'invasion.

Cet acte d'invasion est éprouvé différemment par les entités responsables de la production et de la réception de la traduction. Certaines n'ont généralement pas conscience du rôle de la traduction dans le contact entre cultures. C'est le cas du public, qui consomme la plupart des fois sans trop se poser de questions sur ce qu'on lui offre à lire (ou à entendre) et souvent des traducteurs, qui travaillent avec des délais courts, ayant, par conséquent, peu de disponibilité pour réfléchir aux conséquences de leur activité. Même lorsqu'il s'agit de textes littéraires traduits, dont l'origine étrangère est généralement plus visible et plus assumée, ceux-ci sont conçus comme un moyen d'avoir accès à une littérature étrangère tout court plutôt qu'à une autre culture. La traduction fait pourtant connaître, voire accepter et peut-être même adopter - des traits de langue, des façons de concevoir le monde et des traditions différentes de celles du pays d'arrivée.

Par contre, deux autres intervenants dans ce système semblent très conscients de ce pouvoir de la traduction : les États contemporains, qui agissent souvent comme des agents littéraires et les chercheurs sur la traduction, qui ont, depuis les années quatre-vingt, étudié de façon systématique le rôle de la traduction dans le contact entre cultures.

Ce travail se veut un exercice de réflexion sur le rôle de la traduction en tant que moyen d'invasion culturelle dans les rapports entre la France et le Portugal à l'époque contemporaine. Après une tentative de saisir la position de ces deux États et de ces deux cultures par rapport à la traduction, l'attention y portera sur des exemples concrets, pris de deux romans contemporains, História do cerco de Lisboa, de José Saramago et Je m'en vais de Jean Echenoz, et de leurs traductions en français et en portugais respectivement.

\section{L'État, ce mécène contemporain}

Les États contemporains envisagent de plus en plus la traduction comme un moyen essentiel de divulgation de la culture nationale. La promotion de la littérature nationale à l'étranger dépend de la présence dans les grands salons du livre et de l'aide - généralement financière - à la traduction.

Au Portugal, I'Instituto Camões (qui appartient au Ministères des Affaires Étrangères) a un rôle important dans ce domaine. II offre, chaque année, des "aides à l'édition à 
l'étranger". Les langues d'arrivée sont choisies selon les objectifs de l'organisation, établis chaque année. En 2011, cet institut appuiera surtout la publication d'ouvrages en langue serbe et dans des langues asiatiques. En 2010 la priorité a été de traduire en langue slovène et dans des pays de langue arabe, l'institut ayant appuyé la traduction de quinze ouvrages ${ }^{1}$.

Ceci dit, au Portugal, c'est la "Direcção Geral do Livro e das Bibliotecas" (qui appartient au Ministère de la Culture portugais) qui devient la principale source d'aide à la traduction, avec son "programme de soutien à la traduction". Elle attribue, aux maisons d'édition étrangères qui acceptent de publier des auteurs portugais, des subsides qui assurent le plus souvent près de la moitié des coûts de la traduction, ayant appuyé, à ce jour, la publication de 1.586 ouvrages. En 2010, l'aide à la traduction a correspondu à 156.681,00 Euros et a permis la publication de 110 ouvrages, dans un ensemble de vingtcinq pays, l'Italie et l'Espagne étant ceux où un plus grand nombre d'ouvrages portugais a été traduit, suivis des Pays Bas, de la Suède et de la France ${ }^{2}$. La vérité est que la plupart des livres portugais publiés à l'étranger le sont grâce à cette aide financière.

En France, le Centre National du Livre (un organisme du Ministère de la Culture et de la Communication) est le principal mécène pour ce qui est des traductions. Selon le rapport sur le soutien du livre français à l'étranger, fait, à la demande du ministère de la culture et de la communication, par Marc-André Wagner et Olivier Poivre d'Arvor (2009: 5) " [l]a France mobilise aujourd'hui des moyens significatifs (10 M€) et un dispositif très complet de soutien au livre français et à la création littéraire française à l'étranger". La France attribue ainsi une somme de soutien à la traduction beaucoup plus grande que le Portugal. De même, les modalités d'action du Centre National du Livre sont plus variées que celles de ses congénères portugais. Le premier groupe d'initiatives est composé de trois programmes d'aide directe aux traducteurs: les bourses de séjour (en France) sont attribuées à des traducteurs étrangers pour qu'ils y mènent un projet de traduction d'un ouvrage français ; la " fabrique des traducteurs " permet à de jeunes traducteurs littéraires du et pour le français de mener un projet de traduction et d'être en contact avec des traducteurs expérimentés ; et les "crédits de traduction" sont des subventions pour compenser un traducteur qui travaille sur un ouvrage dont la traduction est considérée difficile et qui n'est pas suffisamment bien payé par la maison d'édition. Un second ensemble d'initiatives contemple une aide aux éditeurs. Ainsi, on attribue des subventions à des maisons d'édition étrangères pour la

\footnotetext{
${ }^{1}$ Trois ouvrages au Maroc, deux en France, deux en Ukraine et un seul ouvrage en Espagne, Hongrie, Israël, Royaume-Uni, République Chèque, Romanie et Suède (Voir http://instituto-camoes.pt/images/stories/pdf/pae.pdf) ${ }^{2}$ Les autres pays étant l'Albanie, Andorre, l'Allemagne, la Bulgarie, la Croatie, le Danemark, les États Unis, la Slovénie, l'Egypte, la Grèce, Israël, la Lettonie, la Lituanie, le Mexique, la Pologne, le Royaume Uni, la Romanie, la Serbie, la Suisse et la Venezuela.

(Voir http://www.iplb.pt/sites/DGLB/Portugues/livro/divulgacaoEstrangeiro/Documents/internacional1.pdf)
} 
traduction d'ouvrages français à l'étranger, prenant charge de la moitié des coûts de la traduction, un des critères de sélection des candidats étant la rareté de la langue ${ }^{3}$.

Il est évident que la traduction est envisagée par les États portugais et français comme une question politique importante et qu'ils portent une attention spéciale à la publication dans des langues périphériques, privilégiant de la sorte la diversité culturelle. La grande différence entre ces deux pays est que la France a un projet plus complexe et financièrement beaucoup plus puissant que le Portugal.

Données statistiques et traditions de traduction

L'absence généralisée de données disponibles sur le nombre de traductions publiées dans chaque pays et leurs pays d'origine est un indice du manque d'attention qui prévaut encore - notamment en Europe - par rapport au rôle de la traduction dans l'échange culturel.

Au Portugal, les seules informations que l'on peut trouver sur la circulation d'ouvrages en traduction sont celles que l'on peut déduire des études sur le marché du livre. En France, à l'inverse, après les efforts faits, tant par Livres Hebdo que par Electre, pour répertorier le nombre de livres étrangers publiés, les chercheurs ont pris cette tâche en charge. Le résultat le plus visible en est la publication de l'ouvrage dirigé par Gisèle Sapiro (2008: 5) qui envisage la traduction comme "vecteur des échanges culturels internationaux " et qui a comme point de départ une enquête sur la circulation de traductions de et pour le Français entre 1980 et 2004. Sapiro (2008: 397) conclut que, malgré la domination de l'anglais dans l'univers de la traduction en France, dans le secteur spécifique des livres de “ rotation lente " - dans lequel peuvent être classés les romans d'Echenoz et de Saramago, puisqu'ils correspondent à une littérature réputée de qualité - il y a une diversification des langues d'origine, l'anglais n'y ayant pas une place aussi importante.

Des efforts commencent aussi à être faits pour répertorier et étudier les données quantitatives et qualitatives de la traduction au niveau européen. Ainsi, l'auteur autrichien Rüdiger Wischenbart $(2008,2009,2010)$ a été responsable de trois études sur les publications de traductions en Europe. À noter que ces études n'incluent guère de données sur le Portugal. Pour ce qui est de la France, on y révèle que, depuis 1998, le nombre de traductions publiées a augmenté et qu'elle est, de nos jours, le pays européen qui publie le plus grand nombre de traductions: près de 10.000 traductions par an (dont soixante-dix pourcent de l'Anglais). Dans cette étude, on caractérise la France comme un pays qui aime bien divulguer sa culture mais qui sélectionne avec attention ce qu'il reçoit.

\footnotetext{
${ }^{3}$ A noter que le Centre National du Livre accorde aussi des subventions pour la traduction d'ouvrages étrangers en Français (Voir http://www.centrenationaldulivre.fr/?-Aides-aux-publications-).
} 
Le Portugal diffère aussi de la France dans ce que l'on pourrait appeler la " tradition de traduction ". Cette expression est le titre donné à une partie importante de l'encyclopédie Routledge sur les études de traduction, dont Mona Baker (2001) est l'éditeur, consacrée précisément à la place qu'a la traduction dans différents pays. Dans cette section de l'encyclopédie, sont prises en considération des données sur l'histoire de la traduction dans chaque pays, la recherche qui y a été menée sur ce domaine, les auteurs (critiques et théoriciens sur la traduction) les mieux connus, ainsi que les traducteurs les plus renommés et l'offre de formation dans le domaine de la traduction. L'encyclopédie inclut des informations sur trente-deux pays, dont la France, évidemment, mais aussi des pays qui n'ont pas une longue tradition d'études sur la traduction, comme la Turquie, la Bulgarie et le continent africain. Or, le Portugal ne compte pas parmi ces pays. En effet, malgré l'effort de quelques chercheurs ${ }^{4}$, une histoire de la traduction au Portugal n'a toujours pas été produite et ce qui y prévaut, comme recherche sur la traduction, ce sont des études de cas.

La France, au contraire du Portugal, compte des siècles de réflexion sur la traduction. Celle-ci inclut des notions (comme "les belles infidèles"), et des noms (Étienne Dolet, Georges Mounin, Antoine Berman) incontournables dans l'histoire de la traduction occidentale. Les ouvrages publiés sur cette thématique abondent, ainsi que les journaux et revues, et l'Institut Supérieur d'Interprétation et de Traduction, ainsi de l'ESIT (de l'Université Sorbonne Nouvelle) sont des institutions ayant une réputation internationale dans la formation de traducteurs et de chercheurs sur cette thématique.

Ce domaine est donc celui où il y a une plus grande disparité entre le Portugal et la France en ce qui concerne la traduction. L'absence d'une tradition forte d'étude et de formation dans le domaine de la traduction au Portugal a d'importantes conséquences sur la façon dont la traduction y est envisagée et pratiquée.

\section{Saramago en France et Echenoz au Portugal}

Les deux auteurs choisis sont des romanciers contemporains qui produisent une littérature réputée de qualité dans leurs pays d'origine. Leur travail appartient à ce que Bourdieu (1998:244) nomme la "production à cycle long”, une littérature qui doit attendre que le lecteur acquière la capacité de la lire et qui ne trouve vraiment sa place dans le champ littéraire au sein duquel elle a été créée que quelques années après la première publication, notamment suite à l'activité des critiques littéraires et de l'école.

Pour ce qui est de Jean Echenoz, il publie chez Les Éditions de Minuit, une maison d'édition orientée vers un public cultivé, qui s'est spécialisée dans une littérature de qualité,

\footnotetext{
${ }^{4}$ Notamment celui de Carlos Castilho Pais (1997), qui a fait un effort de commencer une histoire de la traduction au Portugal, mais dont le seul volume publié ne contemple que le seizième et le dix-neuvième siècles.
} 
souvent d'avant-garde. Bien que cet auteur n'ait commencé à publier que dans les années quatre-vingts, il a déjà une place centrale dans le champ littéraire français. En effet, il a reçu le prix Gouncourt en 1999 (pour son roman Je m'en vais) et ses ouvrages sont étudiés au lycée et à l'université, étant traduits en une trentaine de langues. Toute étude sur la littérature française du vingtième siècle le présente comme un auteur incontournable.

Quant à José Saramago, il publie au Portugal depuis les années cinquante. En 1995, il reçoit le Prémio Camões, le plus important prix littéraire portugais et, trois ans plus tard, le prix Nobel de Littérature. La plupart de ses textes sont publiés chez Editorial Caminho, une maison d'édition qui publie des auteurs portugais contemporains que l'on peut aussi classer dans la "production à cycle long ", comme Sophia de Mello Breyner Andresen e Mário de Carvalho. Il est étudié dans l'enseignement officiel portugais et il est un des auteurs portugais le plus traduit.

Echenoz et Saramago correspondent ainsi à ce que Bourdieu (1998: 358) nomme "l'avant-garde consacrée ", ayant acquis, après des années de travail, une position centrale dans le champ littéraire d'origine. II faut cependant tenir compte des différences entre ces deux auteurs et les champs littéraires que la traduction met ici en contact. En effet, comme le montre Alvaro Manuel Machado (1984: 7), l'influence de la littérature française sur la littérature portugaise est visible depuis toujours, bien qu'elle soit plus manifeste pendant certaines périodes, notamment au dix-neuvième siècle. Ce rapport n'est pourtant pas réciproque, l'influence de la littérature et de la culture portugaise sur la française étant infime. Ceci dit, Saramago a une position plus centrale dans le canon portugais, voire l'occidental, qu'Echenoz dans le français, puisqu'il a reçu le prix Nobel en 1998.

Cela explique probablement que treize romans de Saramago, sur un total de seize, aient été traduits en France ${ }^{5}$. Deux indices semblent suggérer que des efforts ont été faits pour que la version traduite de ces romans soit de qualité. Le premier est que la plupart des textes ont été publiés par une des plus prestigieuses maisons d'édition françaises - Les Éditions du Seuil -, qui a précisément la réputation de ne publier que de la littérature de qualité, dirigée à un public cultivé. Le second est que ces textes ont été traduits par Geneviève Leibrich, une traductrice expérimentée, qui est aussi responsable de la version française des romans de Lídia Jorge et d’António Lobo Antunes, deux renommés auteurs

\footnotetext{
${ }^{5}$ En voici les titres et les dates de publication au Portugal et en France:

Manual de pintura e caligrafia (1977) - Manuel de peinture et de calligraphie (2000); Memorial do convento (1982) - Le Dieu manchot (1987); O ano da morte de Ricardo Reis (1984) - L'Année de la mort de Ricardo Reis (1988); A jangada de pedra (1986) - Le Radeau de pierre (1999); História do cerco de Lisboa (1989) - Histoire du siège de Lisbonne (1992); O Evangelho segundo Jesus Cristo (1991) - L'Évangile selon Jésus-Christ (1993); Ensaio sobre a cegueira (1995) - L'Aveuglement (1997); Todos os nomes (1997) - Tous les noms (1999); A caverna (2000) - La Caverne (2002); O homem duplicado (2002) - L'Autre comme moi (2005) ; Ensaio sobre a lucidez (2004) - La lucidité (2006) ; As intermitências da morte (2005) - Les intermittences de la mort (2008); $A$ viagem do elefante (2008) - Le voyage de l'éléphant (2009).
} 
portugais contemporains ${ }^{6}$. À remarquer aussi que les romans de Saramago étaient déjà traduits en France avant le Nobel, mais qu'après 1998 les traductions sont publiées deux ans en moyenne après la publication au Portugal, tandis qu'avant le Nobel le délai en était de quatre ou cinq ans.

Pour ce qui est de Jean Echenoz, il a écrit douze romans, dont quatre seulement sont traduits en portugais (bien que huit soient traduits en anglais, dix en espagnol, dix en allemand et dix en Italien) ${ }^{7}$. Son avant-dernier roman - Courir - a même déjà été publié au Brésil, mais pas au Portugal. Qui plus est, les dates de publication des traductions portugaises font preuve d'une grande irrégularité et d'un grand écart par rapport à la date de publication des textes français. Aussi, ces quatre romans ont été traduits par trois traducteurs différents, dans trois petites maisons d'édition portugaises ${ }^{8}$. Ces données montrent, à elles seules, que le système éditorial portugais n'a pas investi dans la publication d'Echenoz au Portugal, bien que son travail soit si bien reçu en France et à l'étranger.

Ces différences dans la réception des deux romanciers dans les pays d'arrivée n'est pas sans conséquences. En effet, si l'on accepte le présupposé que la sélection des textes à traduire détermine le canon des littératures étrangères dans le système d'arrivée (Venuti, 1998: 67), on conclut que Saramago est très probablement perçu en France comme un des auteurs les plus importants de la littérature portugaise contemporaine, tandis qu'au Portugal Echenoz est presque méconnu. II est au centre du polysystème littéraire français et dans la périphérie du polysystème de la littérature traduite en portugais.

Stratégies de traduction et visibilité de l'Autre

Après les entités qui ont le pouvoir de choisir les ouvrages à traduire (et, par conséquent, ceux à exclure), les traducteurs sont ceux qui ont le plus d'influence sur le processus d'invasion culturelle, par la sélection qu'ils font des stratégies de traduction. En effet, celles-ci montrent ou cachent l'Autre, dans la mesure où elles peuvent effacer ou exhiber les références culturelles étrangères du texte de départ. Ceci dit, le traducteur n'est pas isolé, il appartient à ce qu'Itamar Even Zohar (2000) appelle un polysystème, un système complexe et hétérogène, comportant des agents et des traditions qui vont déterminer la façon dont le traducteur conçoit son travail. En effet, Even Zohar (2000 : 197) montre comment non seulement la poétique qui prévaut dans la littérature d'un pays détermine la façon dont un texte y est traduit, mais aussi que, lorsque le texte traduit est

\footnotetext{
${ }^{6} \mathrm{O}$ ano da morte de Ricardo Reis et $A$ jangada de pedra ont été traduits par Claude Fage et Memorial do Convento a été publié chez Métailié et Albin Michel.

${ }^{7}$ Les grandes blondes (1995) - As grandes louras (2004) ; Un an (1997) - Un ano (2000) ; Je m'en vais (1999) Vou-me embora (2000) ; Ravel (2006) - Ravel (2007).

${ }^{8}$ Lurdes Júdice, Manuela Torres et Armando da Silva Carvalho en ont été les traducteurs et Terramar, Âmbar et Sextante en ont été les maisons d'édition.
} 
introduit dans un polysystème littéraire plus ancien ou plus puissant, les stratégies de traduction auront tendance à adapter le texte étranger aux normes du système d'arrivée.

Or, depuis les premières réflexions sur la traduction - depuis Cicéron et Saint Jérôme - la notion de fidélité est au centre de la discussion sur la traduction et des soucis des traducteurs. Les traductologues contemporains ont - grâces notamment aux études menées par Susan Bassnett (1998) et André Lefevere (2003) - repris cette question et l'on repensée en l'articulant avec la dimension politique et culturelle de la traduction, dans une perspective moins prescriptive et plus attentive aux conséquences qui adviennent de l'adoption de la fidélité au texte, à l'auteur, à la culture ou au lecteur et des stratégies de traduction qui en découlent. Antoine Berman (2005) et Lawrence Venuti (1998) sont deux des auteurs qui ont souligné - voire critiqué - la tendance qu'a la traduction en général (et certaines traditions de traduction spécifiques) à l'effacement de traits culturels, à être fidèle au lecteur plutôt qu'à la culture de départ. Ils soutiennent même que la traduction doit maintenir un ton d'étrangeté, y compris en termes linguistiques, qu'elle doit avoir pour but premier de souligner la présence du texte étranger, pour que la culture de l'Autre soit perçue en tant que telle. À l'autre extrême de l'échelle sont des traditions de traduction de pays linguistiquement plus puissants comme les Etats-Unis où la tendance générale est à préparer un texte facilement déchiffrable pour le lecteur, ce qui conduit à l'effacement de tout ce qui est différent, étranger.

Pour ce qui est des traductions en étude, aucun doute ne subsiste pour le lecteur ni sur leur statut de traduction, ni sur leurs pays d'origine, des informations que les quatrièmes de couverture des deux ouvrages soulignent d'ailleurs à plusieurs reprises. La même attitude semble déterminer la plupart des stratégies de traduction adoptées par les traductrices, dont le principal effet est de faire connaître les cultures de départ.

Ainsi, dans Histoire du Siège de Lisbonne, un grand nombre des solutions trouvées par la traductrice soulignent l'origine portugaise du texte, comme dans l'exemple suivant, où il y a explicitation de l'origine du texte, par rapport à la version qu'en fournit Saramago : " fundas baleares, ou fundíbulos " (Saramago, 1989:33) est traduit par "frondes lance-balles ou fundas baleares en portugais qu'on appellera aussi fundíbulos " (Saramago, 1992: 33)

En général, les repères culturels portugais sont repris par la traduction, qu'il s'agisse d'un renvoi littéraire ${ }^{9}$, d'une référence historique ${ }^{10}$, ou même de conventions culturelles

\footnotetext{
${ }^{9}$ Lorsque survient dans le texte une référence intertextuelle à un ouvrage d'un auteur portugais peu connu à l'étranger, la traductrice non seulement maintient la référence intertextuelle - imaginons que Romeiro n'ait pas réagi à la curiosité fatale de l'écuyer Telmo " (Saramago, $1992: 87$ ) - mais elle la rend explicite en indiquant le nom de l'auteur et le titre dans une note de bas de page.

${ }^{10}$ Comme celle à Viriato (Saramago, $1992: 201$ ), que la traductrice complémente, à nouveau, par une note de bas de page, indiquant le rôle de ce personnage dans l'Histoire du Portugal.
} 
comme l'usage particulier de titres de civilité ${ }^{11}$. La traductrice y a même très souvent recours à des notes de bas de page - une stratégie qu'Eco (2003:95) considère être une marque de l'échec du traducteur - pour expliciter des références culturelles moins facilement déchiffrables pour le lecteur francophone ou alors essentielles pour la compréhension du récit. Tout traducteur évite de placer des notes de bas de page dans un roman, car celles-ci imposent une pause dans la lecture, pouvant de la sorte interrompre ce que Shaeffer (1999: 180) nomme l'immersion fictionnelle. Or, la traductrice du roman de Saramago en utilise huit. En effet, la note de bas de page est souvent la seule option lorsque le but est de garder une référence culturelle que l'on prévoit ne pas être facilement déchiffrée par le lecteur, mais qu'il s'avère impossible de remplacer par une expression de la langue d'arrivée.

Ce respect des éléments spécifiques de la culture portugaise qui persiste dans la plupart du texte n'est plus visible lorsque les références portent sur la gastronomie portugaise. Les solutions trouvés par la traductrice sont alors, en général, moins précises et remplacent souvent la référence culturelle soit par une référence de la cuisine française cela explique comment les "tostas mistas" (Saramago, 1989: 131) deviennent des “croque-monsieur" (Saramago, $1992:$ 130) et les “jesuítas" (Saramago, $1989: 62)$ des "mille-feuilles" (Saramago, $1992: 63$ ) -, soit par des expressions plus générales, comme lorsque "saucisson" (Saramago, $1992: 38)$ remplace "chouriço " (Saramago, $1989:$ :38) et "gâteaux à la crème" (Saramago, 1992 : 63) remplace "pastéis de nata" (Saramago, 1989 : 62). Le changement d'attitude de la part de la traductrice de Saramago semble correspondre, non à un propos d'effacer la culture portugaise du texte, mais plutôt à une distinction entre ce qui est envisagé comme un élément culturel portugais important et ce qui est perçu comme un détail sans importance. Ceci dit, les "jesuítas ", les "pastéis de nata" et le "chouriço" sont des symboles de la culture portugaise aussi connus et chéris par les portugais que Viriato.

La traduction française du roman de Saramago maintient ainsi, en général, les renvois que l'auteur y fait à la culture portugaise et permet, sans poser trop d'obstacles à la compréhension générale du texte, au lecteur francophone qui le souhaite de mieux connaître certains aspects de la culture portugaise. Elle constitue une exception à cette règle énoncée par Even Zohar selon laquelle une culture plus ancienne et/ou plus puissante aura tendance à produire des traductions qui effacent les caractéristiques spécifiques d'une culture envisagée comme étant moins puissante.

Vou-me embora, la version portugaise du roman d'Echenoz, est aussi ponctuée de références à la culture française. C'est le cas de renvois à l'architecture typiquement

\footnotetext{
${ }^{11}$ La traductrice décide de maintenir l'appellation d'un des personnages centraux, qui inclut un titre de civilité "la doutora Maria Sara ", - tout en expliquant, en note de bas de page, qu'il s'agit d'une " [a]ppellation à la fois respectueuse et familière d'une personne qui possède un titre universitaire" (Saramago, $1992: 100)$.
} 
parisienne ("o típico estilo Haussman" (Echenoz, 2000: 21), "os HLM" (Echenoz, 2000 :55), " os edifícios Napoleão II" (Echenoz, 2000 :109), à des magasins qui font partie de la vie de tous les jours en France (comme le "prisunic" (Echenoz, $2000: 140$ ) et le "Bricorama" (Echenoz, 2000 :93) ou alors à des sigles facilement reconnues par le lecteur français ("a Frac" (Echenoz, 2000 :151) "a SNCF" (Echenoz, 2000 :43), que la traduction portugaise reproduit, ne complémentant l'indication par des notes de bas de page que rarement ${ }^{12}$. Le grand nombre de points de repères géographiques en France, typique de l'écriture de cet auteur ${ }^{13}$, est aussi repris dans la traduction portugaise. D'ailleurs, les mots " rue ", " pont ", " avenue " ou " boulevard " n'y sont pas traduits lorsqu'ils précèdent le nom d'un endroit. Ces options de traduction souviennent constamment le lecteur de l'origine française du roman. Qui plus est, un grand nombre de mots français ont été retenus dans la traduction, alors que des équivalents portugais existent et auraient pu avoir été choisis. C'est de cas de "strapontin"14 (Echenoz, 2000 : 64), "démarches" (Echenoz, 2000 :110), décor (Echenoz, $2000: 114$ ), panne (Echenoz, $2000: 144)$ et "soutien-gorge" (Echenoz, $2000: 151)$.

La traduction portugaise de Je m'en vais adopte ainsi des stratégies de traduction qui produisent un effet d'étrangeté, tel que le définissent Berman et Venuti. Elle exige du lecteur qu'il fasse un effort de lecture supplémentaire, parfois même qu'il ait des connaissances de la langue française, ce qui peut sembler un peu contradictoire, puisqu'un lecteur qui connaisse le sens de "démarches " et de " panne " n'aura probablement pas besoin de lire le roman dans sa version traduite.

\section{Des rapports de forces inattendus}

Bien que le Portugal soit un grand consommateur de traductions et que la France soit, en ce qui concerne les organisations qui encouragent la traduction et leur capacité financière, beaucoup plus puissante que le Portugal, l'œuvre d'Echenoz n'est pas un envahisseur puissant, car elle n'est pas systématiquement traduite en Portugais. Cet auteur et son univers fictionnel sont, par conséquent, très mal connus au Portugal. Ceci dit, dans le cas spécifique de la traduction de Je m'en vais, les conditions sont remplies pour qu'il y ait "invasion culturelle", puisque la culture et la langue française y ont une forte présence. L'absence d'un projet éditorial consistant autour de l'œuvre de cet auteur - visible dans la variation de maison d'édition, de traducteur et de délai dans la publication des traductions -

\footnotetext{
${ }^{12}$ De la liste présentée, seuls les HLM sont accompagnés d'une note de la traductrice qui explique de quoi il s'agit (Echenoz, $2000: 55)$.

${ }_{13}$ A noter que les repères géographiques jouent un rôle crucial dans les textes de cet auteur, que celui-ci caractérise précisément comme des "romans géographiques". Cette notion a d'ailleurs été reprise par Jerusalem (2005) pour expliquer les spécificités des romans d’Echenoz.

${ }^{14}$ Traduit, pourtant, dans une note de la traductrice, comme "assento de dobradiça " (Echenoz, $\left.2000: 64\right)$.
} 
diminue toutefois le pouvoir d'influence que cette traduction aurait pu avoir sur le public portugais.

A l'inverse, le travail de Saramago est systématiquement traduit en Français, généralement par la même traductrice et dans une des plus importantes maisons d'édition française. Dans Histoire du siège de Lisbonne, la culture portugaise résiste au texte français, sans doute grâce à l'effort de la traductrice, et a de bonnes chances de pénétrer dans la culture française. S'il est vrai qu'en général la traduction contribue à la construction de l'image que l'on a de l'Autre, Saramago, son écriture, son imaginaire, et l'histoire et la culture qu'il promeut font sûrement partie de ce que le lecteur français imagine être le Portugal.

Ces deux exemples semblent suggérer que le traducteur et ses stratégies de traduction n'ont pas le pouvoir à eux seuls de déterminer si un texte fonctionnera comme une invasion culturelle. Pour qu'il y ait un vrai impact de la culture étrangère sur la culture nationale il faut aussi que d'autres entités, comme des organismes de l'État et/ou les maisons d'édition, fassent la promotion du projet. 


\section{Bibliographie}

BAKER, Mona (ed.) (2001). Routledge encyclopedia of translation studies. London-New York: Routledge.

BASSNETT, Susan, LefEVERE, André (1998). Constructing cultures: essays on literary translation. Clevedon : Multilingual Matters.

BERMAN, Antoine (2000). "Translation and the trials of the foreign". In: Lawrence VENUTI, éd., The translation studies reader. London-New York: Routledge, pp.284-297.

BOURDIEU, Pierre (1998). Les règles de l'art. Paris : Éditions du Seuil.

ECHENOZ, Jean (1999). Je m'en vais. Paris : Minuit.

Echenoz, Jean (2000). Vou-me embora, traduit par Manuela Torres, Lisboa: Terramar.

Eco, Umberto (2003). Dire quasi la stessa cosa. Esperienze di traduzione. Milano: Bompiani. EVEN ZOHAR, Itamar (2000) "The position of translated literature within the literary polysystem". In: Lawrence VENUTI (éd.) The translation studies reader. Londres - New York : Routledge.

JeRUSALEM, Christine (2005). Jean Echenoz: géographies du vide. Paris : Publications de I'Université de Saint-Etienne.

LefeVere, André (2003). "Introduction”. In : André LefEVERE (éd.) Translation, history, culture. Londres, New York: Routledge.

MACHADO, Álvaro Manuel (1984). O "francesismo " na literatura portuguesa. Lisboa: Instituto de Cultura e Língua Portuguesa.

PAIS, Carlos (1997). Teoria diacrónica da tradução portuguesa. Antologia (séc.XV-XX). Lisboa: Universidade Aberta.

SARAmago, José (1989). História do cerco de Lisboa. Lisboa: Caminho.

SARAMAGo, José (1992). Histoire du siège de Lisbonne, traduit par Geneviève Leibrich, Paris : Éditions du Seuil.

SCHAEFFER, Jean-Marie (1999). Pourquoi la Fiction ? Paris: Seuil.

VENUTI, Lawrence (1998). "The formation of cultural identities", In: Lawrence VENUTI. The scandals of translation. Towards an ethics of difference. London, New York: Routledge. WAGNER, Marc-André, PoIVRE D'ARVOR, Olivier (2009). Quelles perspectives pour la politique publique de soutien au livre français à l'étranger?: Propositions pour une stratégie concertée des acteurs publics. Centre National du Livre. [disponible le 28/04/11] $<U R L$ :http://lesrapports.ladocumentationfrancaise.fr/BRP/094000466/0000.pdf>. WISCHENBART, Rüdiger (2008). Diversity Report 2008. An overview and analysis of translation statistics across Europe: facts, tends, patterns. [disponible le 28/04/11]

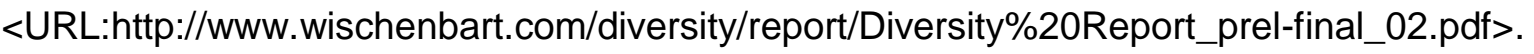


WISCHENBART, Rüdiger, KovAC, Miha (2009). Diversity report 2009. Cultural diversity in translations in books: mapping fiction authors across Europe. [disponible le 28/04/11]

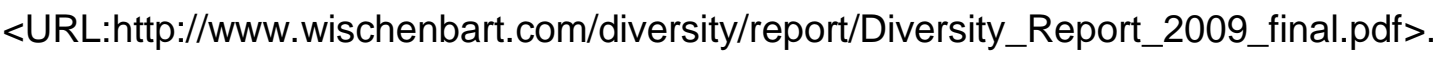
WISCHENBART, Rüdiger, KOVAC, Miha (2010). Diversity report 2010. Literary translation in current European book markets. An analysis of author, languages, and flows. [disponible le 28/04/11] <URL:http://www.wischenbart.com/diversity/report/Diversity-Report_2010.pdf>. 
